\title{
The Analysis of Electromagnetic Radiation at Destruction of Structural Materials
}

\author{
Borisov VD* \\ Department of Mechanical Engineering, Teplosnabzhenie, Belovo, Russia
}

\begin{abstract}
The processes occurring in the structural material under load, leading to the formation of electromagnetic radiation, are briefly considered. It is shown that there is a relationship between the duration of the anterior front of the electromagnetic radiation pulse and the length of the crack responsible for the formation of this pulse. The task of the analysis of electromagnetic radiation signals is to establish quantitative relations between the parameters of the signal and the parameters of the crack responsible for the formation of this signal. The development of the method of spectral-temporal analysis in this application is briefly described. The application of the fractal analysis method for qualitative estimation of the fracture surface size is considered, as well as the relations are introduced: frequency of the spectral component-characteristic size of the crack (length), amplitude of the spectral component - the number of cracks. On the basis of the model, which combines the principles of both linear and nonlinear mathematical physics, the transition to the quantitative evaluation of crack parameters by electromagnetic radiation signals is performed. The phenomenon called "High-Frequency Trace", the main property of which is inversely proportional, almost linear dependence of the number of cracks on their size, was found. Some parts of these dependencies have logarithmic scale invariance.
\end{abstract}

Keywords: Deformation; Electromagnetic radiation; Time-andspectrum analysis; Fractal analysis; Time-and-spatial type of the analysis; Self-similar structure

\section{Literature Review}

\section{Physics of electromagnetic radiation signal generation}

At destruction of construction materials (further on the text simply materials) of various origin (it can be rocks, metals, etc.) electromagnetic radiation (EMR) is formed. The formation of EMR in this case due to the following processes: breaking of inter atomic, inter knots communications, the appearance of free electrons and vacancies, the movement of charges in the peaks and on the shores of the formed cracks. The greatest contribution to these processes is made by the charges moving in tops of cracks, the size of these charges reaches to $90 \%$ of the total charge formed in the course of development of a crack [1]. As it is known [2], there is a communication between the duration of the release of elastic energy and the size of cracks formed under load, which consists in the fact that the time of growth of the longitudinal component of the elastic wave is proportional to the length of the crack. Moreover, this connection is universal and exists on a scale ranging from nanometres to thousands of kilometres. A similar dependence exists for EMR, it consists in the proportionality of the duration of the forward front of the EMR pulse, the length of the crack responsible for the generation of this pulse [3]. Thus, the physics of EMR signal formation determines the fact that the EMR signal spectrum contains information about the parameters of cracks responsible for the formation of this signal.

\section{Problems and methods of the analysis of signal EMR}

In this regard, there is a task that has both purely scientific and practical importance, which is to establish quantitative relations between the parameters of the EMR signal and the parameters of cracks responsible for the formation of this signal. The solution of such a problem allows observe (control) the processes taking place in the destroyed object in real time, for example, in order to control the state of the object or predict the moment of its destruction. In this direction, the author performed the following actions
1. In the development of time-and-spectrum analysis (TSA), the technique of constructing time-and-spectrum tables (TST), allowed to identify the composition of the signal EMR-defined structure. The essence of this technique is as follows. First of all, the visual (approximate) evaluation of the signal parameters, such as maximum amplitude, frequency range, number of extrema, points of transitions through zero, time intervals between them, according to which the selection of the signal fragment for analysis and determination of parameters in the construction of TST. The graphic image of the EMR signal is taken as the initial one, and the sequence of digital samples of the signal with an interval is used for the analysis. The following actions are performed for the selected signal fragment. The sequence of digital signal samples is divided into subsequences with a shift equal to the line spacing TST. The duration of the subsequences is the number of FFT points that will be applied to each subsequence at a later stage. The results of the FFT are recorded in the TST lines according to the time shift. In detail, this technique and the choice of transformation parameters, as well as an example of experimental data processing are presented in time-and-spectrum analysis to study rock failure dynamics [4].

2. Fractal analysis elements are used for qualitative evaluation of the fracture surface formed in the material under load. According to the position of Griffiths fracture theory [5],

*Corresponding author: Borisov VD, Department of Mechanical Engineering, Teplosnabzhenie, Belovo, Russia, Tel: +7 (3822) 653-761; E-mail: victor.borisov@ngs.ru

Received May 02, 2018; Accepted May 13, 2018; Published May 17, 2018

Citation: Borisov VD (2018) The Analysis of Electromagnetic Radiation at Destruction of Structural Materials. J Appl Mech Eng 7: 305. doi:10.4172/21689873.1000305

Copyright: @ 2018 Borisov VD. This is an open-access article distributed under the terms of the Creative Commons Attribution License, which permits unrestricted use, distribution, and reproduction in any medium, provided the original author and source are credited. 
the crack propagation leads to a decrease in the energy of elastic deformations $\mathrm{W}$ (elastic energy) of the body due to its unloading near the crack propagation region, and an increase in its surface energy $U$ as a result of the formation of new surfaces of the body, with the following equation:

\section{$\mathrm{dW} / \mathrm{dt}=\mathrm{dU} / \mathrm{dt}$}

The process of formation of new surfaces is accompanied by emission of elastic waves (acoustic emission (AE) and electromagnetic radiation. AE occurs directly as a result of the release of elastic energy and the propagation of elastic waves on the surface of the material. On the mechanism of formation of EMR mentioned above. Here it should be borne in mind that the crack surface is a complex formation of in heterogeneities of different scale levels, and in the process of formation of this surface generated many EMR pulses (as different scale levels, both in time and amplitude). Therefore, the real EMR signal is the result of superposition of many different signals, and it is almost impossible to single out a single pulse of any scale level from such a signal. Therefore, it is convenient to use the frequency area rather than the time area to represent such a signal. In line with this, if in the time area there is a directly proportional relationship between the duration of the leading edge of the pulse EMR and the length of the cracks $\left(t i=k_{1} l i\right.$ where $\mathrm{k}_{1}$-coefficient of proportionality), responsible for the generation of this pulse, in the frequency area, this corresponds to the inverse relationship between the frequency of a certain spectral component of the signal EMR and the linear size of some cracks responsible for the contribution of this spectral component in the overall frequency spectrum of the signal EMR:

\section{$\left(\mathrm{fi}=1 / \mathrm{k}_{1}\right) \times \mathrm{li}$}

where $\mathrm{k}_{1}$-coefficient of proportionality, fi-frequency of the $\mathrm{i}^{\text {th }}$ spectral component of the EMR signal, li-length of the $i^{\text {th }}$ in homogeneity. EMR signals by their information content are close to secondary electrons in a raster electron microscope, which came out from the surface of the object under study [6]. A significant difference only in that the secondary electrons are reflected by the surface of the material and carry information about this surface, and only indirectly - about the internal processes of deformation. EMR signals are formed by surfaces of formed cracks of different scale levels, which are both on the surface of the sample and in its depth and have in their composition information about the parameters of these surfaces. As it is known $[7,8]$, cracks formed in rocks have self-similar structure. For example, if you look at the fracture of a rock in an electron microscope at different magnification scales, you see about the same picture. This means that the number of in heterogeneities of different sizes, at the appropriate review scale, is approximately constant. In general, the property of selfsimilarity is described by the expression [7]:

\section{$\mathrm{N}=\mathrm{rd}$}

where $\mathrm{N}$ is the number of elements of observation, $\mathrm{r}$-is the coefficient of similarity, d-is the dimensionality of the study area (in the case of fractional values is called fractal). The sequence of actions is as follows. As a result of the application of TSA to the digital image of the EMR signal, the time-and-spectrum tables are constructed, the rows of which correspond to the moments of time, as well as to the load level, and the columns correspond to the frequencies of spectral components. Thus, in each line of TST the spectral image of the electromagnetic radiation signal corresponding to a certain moment of time and a certain stage of the destruction process is presented. Further, considering the relationship between the frequencies of the spectral components of the EMR signal and the size of the cracks responsible for the generation of these spectral components, we transfer the consideration from the frequency region to the spatial area, i.e., to the geometry area. Then, guided by the principle of linear superposition [9], according to which in the nature of the action from the set of elementary sources are formed in the phase, the frequency amplitudes of spectral components are correlated with the number of cracks of this size:

\section{$\mathrm{Afi}=\mathrm{k}_{2} \times \mathrm{NLi}$}

where $\mathrm{k}_{2}$ is the proportionality factor, Afi is the amplitude of the $\mathrm{i}^{\text {th }}$ spectral component, NLi is the number of cracks of the $\mathrm{i}^{\text {th }}$ length corresponding to the $\mathrm{i}^{\text {th }}$ spectral component. Having gone further in our reasoning, we present each line of TST (the state of the destruction process) with a set of dimensions of cracks (frequency of spectral components) and the number of cracks of each size (amplitude of spectral components). Then, for each of the sequences of the number and sizes of cracks that make up the TST lines, the fractal dimension $\mathrm{D}$ is calculated (the Fractal dimension of the object characterizes the degree of filling of space by the object [7]. On that, how much size D is close to size of dimension of a surface, i.e., to two, judge a stage on which there is a process of formation time during which such value $D$ remains, are an indicator of what cracks have taken part in surface and time formation during which there is a given surface. This technique is described in more detail in article [10].

3. Made the transition from qualitative to quantitative estimates of the parameters of cracks according to the signals EMR. This is done on the basis of a model that combines the principles of both linear and nonlinear mathematical physics and is a kind of transformation from time-and-spectrum, in time-and-spatial type of the analysis. The correspondence between parameters of EMR signal and parameters of cracks in the model is based on the following provisions:

(i). Mechanical vibrations (longitudinal, torsional) when considering the simplest case of oscillations with one degree of freedom and electrical vibrations lead to the same differential equation [11]. It also presents the cases of the beats (the result of the interaction of oscillations) between the oscillations of different physical nature mechanical, acoustic, electric. The transformation of one type of oscillation into another occurs through interaction through elements of the environment

(ii). Experimentally, it was found [12] that when an elastic wave with a frequency $\mathrm{v}_{\mathrm{a}}$ passes through the crystal, an electromagnetic signal is excited, in the spectrum of which the frequency $\mathrm{v}_{\mathrm{a}}$ prevails.

Thus, when constructing the author proceeded from the fact that spectral components are present in the EMR spectrum, with frequencies proportional to the characteristic sizes of cracks. This model is described in detail in [13].

\section{Discussion and Conclusion}

In conclusion, let us say that in the study of the EMR signal obtained from the destruction of the sample of marble in the laboratory, by the method described above, a phenomenon called "High-Frequency trace" ("HF trace"), containing areas that have the property of scale invariance. The most interesting feature of the "HF trace" is close to the linear inverse - proportional dependence of the number of cracks on their size. A similar method was used to process the EMR signal obtained from the destruction of the sample of diabase. A rich spectrum of nonlinear oscillations and waves, solitary waves, solitonlike oscillations, in particular kink (topological soliton, dislocation in the Frenkel-Kontorova model [14] was obtained here. 
Citation: Borisov VD (2018) The Analysis of Electromagnetic Radiation at Destruction of Structural Materials. J Appl Mech Eng 7: 305. doi:10.4172/21689873.1000305

Page 3 of 3

\section{References}

1. Alekseev DV, Egorov PV (1993) On the shape of electromagnetic pulses generated by moving cracks, Fiz-Tekh, Probl, Razrab, Polezn, Iskop 6: 3-5.

2. Kuksenko VS, Lyashkov Al, Mirzoev KN (1982) Communication between the sizes of cracks formed under loading and duration of allocation of elastic energy. Dokl Akad Nauk SSSR 264: 846-848.

3. Borisov VD (2005) About a parity of the linear sizes of heterogeneities of microcracks and parametres of signals of electromagnetic radiation. Gorgny Inst UrO RAN pp: 34-37.

4. Borisov VD (2005) Time-and-spectrum analysis to study rock failure dynamics. Fiz-Tekh Probl Razrab Polezn Iskop 4: 49-59.

5. Libovits G (1975) Mathematical bases of the theory of destruction 2: 1.

6. Mir M (1988) Fractals in Physics. Russian translations, Moscow, Russia

7. Mandelbrot BB (1983) The fractal geometry of nature. 2: 1.
8. Feder J (1991) Fractals.

9. Claerbout JP (1981) Fundamentals of geophysical data processing as applied to oil exploration. Library of Congress Cataloging in Publication Data, Blackwell Scientific Publications, USA.

10. Borisov VD (2007) Fractal properties of the spectral characteristics of electromagnetic radiation during fracture of rocks and structural materials. FizTekh Probl Razrab Polezn Iskop 2: 55-69.

11. Hartog JPD (1985) Mechanical vibrations. Dover Publications, USA.

12. Ko B, Yavorovich LV, Fedotov PI (2005) Relationship between the parameters of electromagnetic signals and the electrical characteristics of rocks during acoustic and quasi-static effects. Izv Tom Gos Politekh Univ 308: 1-6.

13. Borisov VD (2018) Determining the parameters of microcracks from thei electromagnetic signals. Prikl Mekh Tekh Fiz 59: 129-137.

14. Frankel $Y$, Kontorova $T$ (1939) On the theory of plastic deformation and twinning. Fiz Zh 1: 137-139. 